\title{
Intraductal papillary neoplasm of the bile duct found incidentally during examination of colonic disease: A case report
}

\author{
Katsuhiro Tomofuji, Yoshihiro Mise, Hiromichi Ito, Takeaki Ishizawa, Yosuke Inoue, Yu Takahashi and Akio Saiura* \\ Department of Gastroenterological Surgery, Cancer Institute Hospital of the Japanese Foundation for Cancer Research, Tokyo, Japan
}

\begin{abstract}
Background: Intraductal papillary neoplasm of the bile duct (IPNB) is a relatively rare bile duct neoplasm in the multistep carcinogenesis pathway towards cholangiocarcinoma. The diagnosis of IPNB is challenging, and its malignant potential means that its prognosis is related to the performance of R0 resection. We experienced a patient with IPNB detected during close examination of colonic disease and subsequently treated surgically.

Case presentation: A 54-year-old man presented to an outside hospital with abdominal pain. Enhanced computed tomography showed wall thickening in the ascending colon and a cystic lesion close to the posterior segmental branch of the bile duct in the liver. Colonoscopy showed diverticulitis and severe stenosis in the ascending colon, with no finding of malignancy. Differential diagnosis of the liver tumor was IPNB, mucinous cystic neoplasm, or metastatic tumor from the colonic disease. The patient underwent hepatectomy and simultaneous right hemicolectomy for severe diverticulitis. Histopathological examination of the liver revealed IPNB with an associated invasive carcinoma.

Conclusions: We report a relatively rare case of IPNB. Although preoperative diagnosis of IPNB remains challenging, complete resection is important for a favorable prognosis.
\end{abstract}

\section{Introduction}

Intraductal papillary neoplasm of the bile duct (IPNB) has recently been proposed as the biliary counterpart of intraductal papillary mucinous neoplasm of the pancreas [1], and can develop within any part of the biliary tree. Early systems classified bile duct tumors on the basis of their ability to produce mucin. IPNB is identified as a premalignant lesion or intraepithelial neoplasm of the bile duct, and has also been described anecdotally as having limited invasive potential, typically involving only cellular atypia, and at most carcinoma in situ $[2,3]$. However, tumors eventually invade into the bile duct wall and progress to invasive cholangiocarcinoma. The clinical manifestations of IPNB include obstructive jaundice and cholangitis, but IPNB can also be detected incidentally in asymptomatic individuals. Early surgery should be performed in all cases of suspected IPNB based on clinical findings and imaging alone, to prevent disease progression [3]. We experienced a surgical case of IPNB detected during close examination of colonic disease.

\section{Case presentation}

A 54-year-old man presented to an outside hospital with abdominal pain. Enhanced computed tomography (CT) showed ascending colonic wall thickening and swollen lymph nodes and a cystic lesion close to the posterior segmental branch of the bile duct in the liver. Colon cancer with liver metastasis was suspected at the previous hospital, and the patient was referred to our institution.

The patient's medical history included an appendectomy at 20 years of age, hypertension, and congenital anomalies of the kidney. He had no history of liver disease.
Laboratory results before surgery were as follows: hemoglobin, 11.3 $\mathrm{g} / \mathrm{dL}$; total bilirubin, $0.2 \mathrm{mg} / \mathrm{dL}$; aspartate aminotransferase, $18 \mathrm{IU} / \mathrm{L}$; alanine aminotransferase, $13 \mathrm{IU} / \mathrm{L}$; alkaline phosphatase, $195 \mathrm{IU} / \mathrm{L}$; and $\gamma$-glutamyl transpeptidase, $22 \mathrm{IU} / \mathrm{L}$. Levels of tumor markers, including alpha-fetoprotein, protein induced by vitamin $\mathrm{K}$ absence, carcinoembryonic antigen, and carbohydrate antigen 19-9 were within normal limits. Viral markers for hepatitis B and C were negative.

Colonoscopy showed chronic ascending colonic diverticulitis and no finding of malignancy. CT and magnetic resonance imaging (MRI) showed a gradually enhanced mural nodule in the cyst located at the proximal posterior segmental branch of the bile duct (Figure 1). T1weighted MRI showed a low-intensity mass. The signals from the cystic lesion were high on T2-weighted images, except for the mural nodules. The cystic lesion was suspected to be a mucin-producing liver tumor, such as a mucinous cystic neoplasm of the liver, IPNB, a hemangioma, or a schwannoma of the liver.

The suspected colon cancer was finally diagnosed as diverticulitis, and the intrahepatic cystic lesion including mural nodules on CT and MRI was considered to be IPNB. Because the patient had a history of repeated diverticulitis with severe stenosis and because

Correspondence to: Akio Saiura, Department of Gastroenterological Surgery, Cancer Institute Hospital of the Japanese Foundation for Cancer Research, 3-831 Ariake, Koto-ku, Tokyo 135-8550, Japan, E-mail: saiura-tky@umin.ac.jp

Key words: Intraductal papillary neoplasms of the bile duct, intrahepatic cystic tumor, bile duct carcinoma

Received: June 26, 2017; Accepted: July 17, 2017; Published: July 19, 2017 

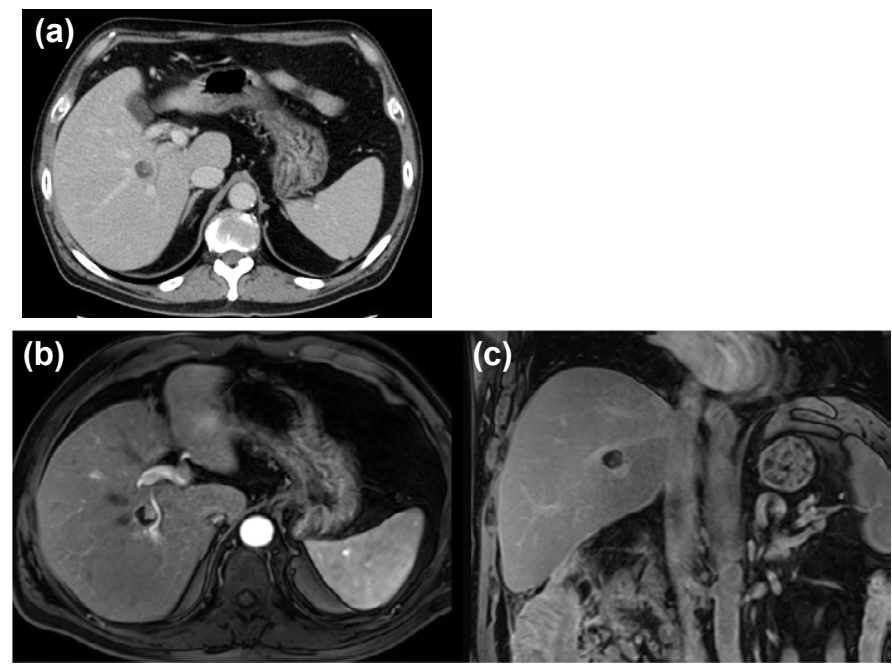

Figure 1. Abdominal contrast computed tomography (CT) and enhanced magnetic resonance imaging (MRI) findings. (a) Contrast-enhanced CT shows a low-density hepatic lesion measuring $1.5 \mathrm{~cm}$ in diameter with mural nodules with contrast-enhanced high signals (a red arrow). (b, c) Contrast-enhanced MRI shows lesion with enhanced nodules located at the proximal part of the posterior bile duct.

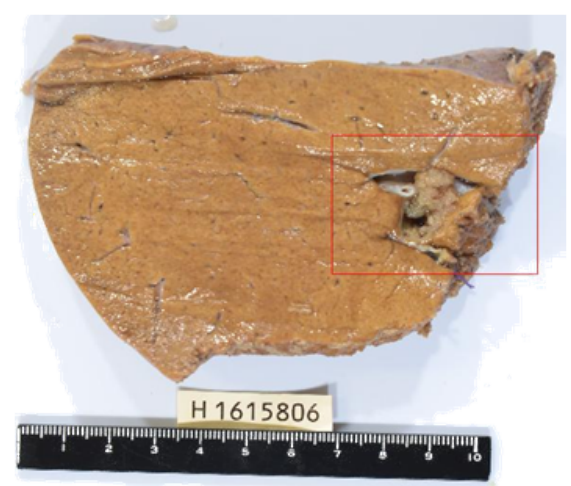

Figure 2. Macroscopic findings of resected specimen. The cut surface of the resected specimen shows papillary nodules from the lining wall of the intrahepatic bile duct and contains mucinous fluid.

IPNB has a potential for malignancy, resection was planned for both involvements. We performed extended posterior right sectionectomy, and hemicolectomy for chronic diverticulitis. The resected cyst was composed of papillary nodules on the wall filled with clear mucus. No recurrent disease was found during the 10-month follow-up period.

\section{Pathological findings}

The surgically resected specimen revealed a tumor in the intrahepatic biliary tree of the right posterior section (Figure 2). The cut surface revealed a mucinous appearance with mucin infiltrating the cyst.

Histopathologically, the tumor contained invasive components. The intraductal component showed papillary growth and extracellular mucin. Part of the tumor invaded through the bile duct wall and formed a cyst (Figure 3 ). The cystic tumor lacked an ovarian-like stroma. MUC1 and MUC2 immunostaining were negative (Figures $4 \mathrm{a}$ and $4 \mathrm{~b}$ ), while MUC5AC displayed diffuse positivity in the invasive and intraductal components (Figure 4c).

On the basis of these findings, the cystic tumor was finally diagnosed as IPNB (invasive adenocarcinoma, gastric type) invading the bile duct

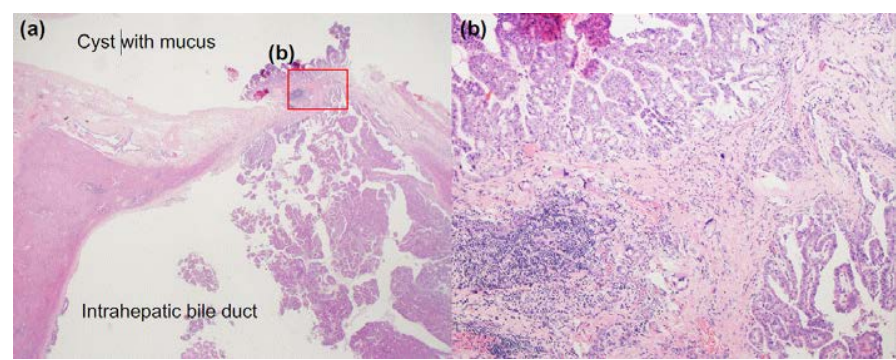

Figure 3. Microscopic features of the tumor. (a) Histopathological findings reveal papillary nodules from the lining wall of the intrahepatic bile duct, and the tumor invades through the bile duct wall and forms a cyst containing secreted mucus. (b) The tumor was composed histologically of an invasive adenocarcinoma with papillary structures invading the stroma.

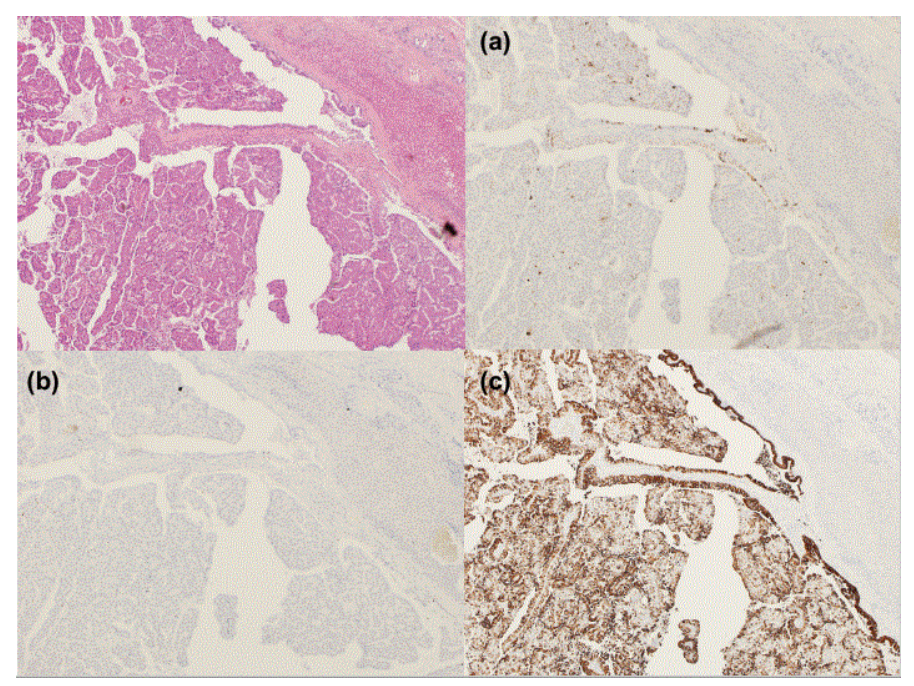

Figure 4. Immunohistochemical characterization. The tumor cells were negative for MUC1 (a) and MUC2 (b), and positive for MUC5A (c).

wall and forming a cyst besides the bile duct of the posterior segment of the right hepatic lobe.

\section{Discussion}

We report a surgical case of IPNB in the liver, found incidentally during close examination of colonic diverticulitis.

Preoperative diagnosis of IPNB is challenging, and the current case demonstrates the incidental radiological detection of a cystic tumor, mimicking colorectal liver metastases. The intrahepatic bile ducts showed no changes such as dilatation on preoperative images. Previous reports have indicated that most IPNB patients present with right hypochondria (35-88.5\%), recurrent acute cholangitis (5-59\%), and obstructive jaundice (20-36\%) [4-6]), but that IPNB may also be detected incidentally in asymptomatic individuals (5-12\%) [3,4]. IPNB remains difficult to differentiate from cholangiocarcinoma and other types of bile duct disease, and may mimic colon cancer metastases on radiological findings [7]. The characteristic CT and MRI findings of IPNB are a dilated and cystic biliary system and multifocal papillary epithelial lesions. In the current case, the large bile ducts around the cystic lesion were unaffected radiologically, and the patient was suspected to have liver metastasis from colon cancer on the basis of thickening of the ascending colonic wall and swollen lymph nodes detected on CT. However, there was no sign of colon cancer on colonoscopy, and the final diagnosis was diverticulitis.

In patients with IPNB, the tumor originates from the intrahepatic 
bile duct and invades through the bile duct wall, and a cystic lesion is formed by mucin secreted by the tumor. In the present case, preoperative images showed no changes such as dilatation of the bile duct, buthistological findings revealed dilatation of the intrahepatic bile duct around the tumor.

IPNB is regarded as a tumor with malignant potential, which can be categorized into one of three groups, according to the degree of malignancy: adenoma (low grade), borderline (intermediate grade), and malignant (carcinoma in situ and high grade). IPNB accounts for $7 \%-38 \%$ of all bile duct carcinomas, and $40 \%-80 \%$ of IPNBs contain invasive carcinomas $[3,6]$. However, IPNB is generally less invasive and has a more favorable outcome than conventional bile duct cholangiocarcinoma. Lee et al. reported a disease-free survival rate of $81 \%$ at 5 years among 58 patients with IPNB who underwent curative resection, with a mean survival period of $60.87 \pm 5.86$ months $[6,8]$. However, patients with invasive tubular carcinoma carry a poorer prognosis than mucinous/colloid carcinoma or IPNB alone. Although it is difficult to make an accurate preoperative diagnosis because of the low incidence of IPNB and the lack of specific clinical manifestations, preoperative and pathological diagnoses are important for determining its prognosis. Kang et al. reported that the accuracy of predicting macroscopic multiplicity based on preoperative radiologic imaging findings was $53.5 \%$, with a false positive rate of $25.8 \%$ and a false-negative rate of $37.7 \%[6,9]$. Patients without distant metastasis are considered for surgical resection [10]. R1 or R2 resection, depth of extraductal invasion, grade of invasion, and lymphovascular invasion have been associated with a poorer prognosis [4]. In the current case, we performed R0 resection, and no lymphovascular invasion was detected. The significant risk of invasive bile duct carcinoma means that early surgical intervention is crucial to achieving a long-term recurrencefree outcome. This case highlights the need to consider IPNB during the differential diagnosis of cystic intrahepatic tumors.

\section{Conclusions}

We are report a relatively rare case of IPNB. Although preoperative diagnosis of IPNB remains challenging, curative resection is the major treatment and an important factor in determining long-term survival.

\section{Declarations}

Ethics approval and consent to participate: Ethical approval was given by the Cancer Institute Hospital of the Japanese Foundation for Cancer Research ethics committee.

Competing interest: The authors declare that they have no competing interests.

Authorship and Contributorship: Katsuhiro Tomofuji: Acquisition of date,interpretation of date, drafting the article, revision, final approval of the version to be published;Yoshihiro Mise: Concept and design of study, revision, final approval of the version to be published; Hiromichi Ito: Final approval of the version to be published;Takeaki Ishizawa: Final approval of the version to be published; Yosuke Inoue: Final approval of the version to be published;Yu Takahashi: Final approval of the version to be published;Akio Saiura: Final approval of the version to be published, agreement to act as guarantor of the work.

\section{References}

1. Lim JH, Zen Y, Jang KT, Kim YK, Nakanuma Y (2011) Cyst-Forming Intraductal Papillary Neoplasm of the Bile Ducts: Description of Imaging and Pathologic Aspects. American Roentgen Ray Society 197: 1111-11120.

2. Takahashi G, Kuroda S, Tashiro H, Kobayashi T, Ishiyama K, et al. (2015) A case of biliary cystic tumor with repeated hemobilia. Surg Case Rep 1: 34.[Crossref]

3. Gordon-Weeks AN, Jones K, Harriss E, Smith A, Silva M (2016) Systematic Review and Meta-analysis of Current Experience in Treating IPNB. Ann Surg 263(4): 656-663. [Crossref]

4. Rocha FG, Lee H, Katabi N, DeMatteo RP, Fong Y, et al. (2016) Intraductal papillary neoplasm of the bile duct: a biliary equivalent to intraductal papillary mucinous neoplasm of the pancreas? Hepatology 56(4): 1352-1360.[Crossref]

5. Tan Y, Milikowski C, Toribio Y, Singer A, Rojas CP, et al. (2015) Intraductal papillary neoplasm of the bile ducts: A case report and literature review. World J Gastroenterol 21: 12498-12504.[Crossref]

6. Wan XS, Xu YY, Qian JY, Yang XB, Wang AQ, et al. (2013) Intraductal papillary neoplasm of the bile duct. World J Gastroenterol 19: 8595-8604.[Crossref]

7. Yamao T, Hayashi H, Higashi T, Takeyama H, Kaida T, et al. (2015) Colon cancer metastasis mimicking intraductal papillary neoplasm of the extra-hepatic bile duct. Int J Surg Case Rep 10: 91-93.[Crossref]

8. Kim KM, Lee JK, Shin JU, Lee KH, Lee KT, et al. (2012) Clinicopathologic features of intraductal papillary neoplasm of the bile duct according to histologic subtype. Am J Gastroenterol 107: 118-125.[Crossref]

9. Kang MJ, Jang JY, Lee KB, Han IW, Kim SW (2013) Impact of macroscopic morphology, multifocality, and mucin secretion on survival outcome of intraductal papillary neoplasm of the bile duct. J Gastrointest Surg 17(5): 931-938.[Crossref]

10. Vibert E, Dokmak S, Belghiti J (2010) Surgical strategy of biliary papillomatosis in Western countries. J Hepatobiliary Pancreat Sci 17: 241-245.[Crossref]

Copyright: (C2017 Tomofuji K. This is an open-access article distributed under the terms of the Creative Commons Attribution License, which permits unrestricted use, distribution, and reproduction in any medium, provided the original author and source are credited. 